\title{
Density field projection analysis in search for WHIM
}

\author{
Lauri Juhan Liivamägi \\ Tartu Obseratory, Tõravere, Nõo vald 61602, Estonia
}

\begin{abstract}
We study the possibilities of using line-of-sight projected luminosity density and filament probability fields to find the best locations in the sky to attempt to observe the elusive Warm-Hot Intergalactic Matter (WHIM). We calculate galaxy luminosity fields on a regular grid and then project those to the plane of sky. Column densities are then found by integrating along multiple lines of sight. We also create the projections of the filament probability fields. The results are presented as sky maps of the potentially suitable areas and the corresponding fractions of sky coverage.
\end{abstract}

Keywords. cosmology: observations, large-scale structure of universe, galaxies: intergalactic medium

\section{Introduction}

At low redshifts $(z<2)$ all observations of the visible matter sum up to half of the expected cosmological mass density of baryons (Nicastro et al. 2008). Large scale structure formation simulations suggest that these missing baryons reside in the form of WHIM in the filamentary cosmic web structure connecting the clusters of galaxies and superclusters (Cen \& Ostriker 2006). At the predicted temperatures of $10^{5}-10^{7} \mathrm{~K}$ and densities $10^{-6}-10^{-4} \mathrm{~cm}^{-3}$ the X-ray emission from WHIM structures is too faint to be detected with current instrumentation. However, the column densities of the highly ionised WHIM metal ions along large-scale structures can reach a level of $10^{15}-10^{16} \mathrm{~cm}^{-2}$, imprinting detectable absorption features on the soft X-ray or UV spectra of background sources. So far, only few tentative observations e.g. at Sculptor Wall (Buote et al. 2009) have been made.

Density fields are standard method to study large-scale structures of the universe. Common approach is to assume a Cox model for the galaxy distribution, where the galaxies are distributed in space according to a inhomogeneous point process with the intensity $\rho(\mathbf{r})$ determined by an underlying random field (see, e.g. Martínez \& Saar 2003). It means that, although expecting certain biases, we expect galaxies to adequately trace the distribution of mass. As filamets can be a very suitable for WHIM detection we also attempt to use the SDSS filament sample created by Tempel et al. (2014).

\section{Density fields}

Data. We carried out the initial analysis on the SDSS DR8 main galaxy sample (Aihara et al. 2011). We used the data from the contiguous 7221 square degree area in the North Galactic Cap (Fig. 1). The sample selection is described in detail in the SDSS DR8 group catalogue paper by Tempel et al. (2012). We calculated the absolute magnitudes of galaxies in the SDSS $r$-band and applied correction for Galactic extinction, $k$-correction and evolutionary correction. The resulting flux-limited galaxy sample contains 576493 


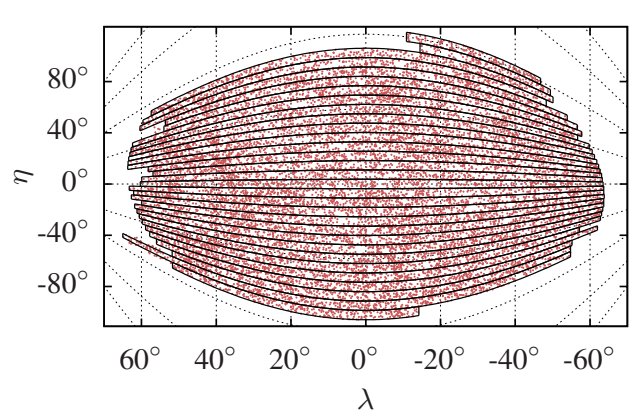

Figure 1. SDSS DR8 main sample footprint.

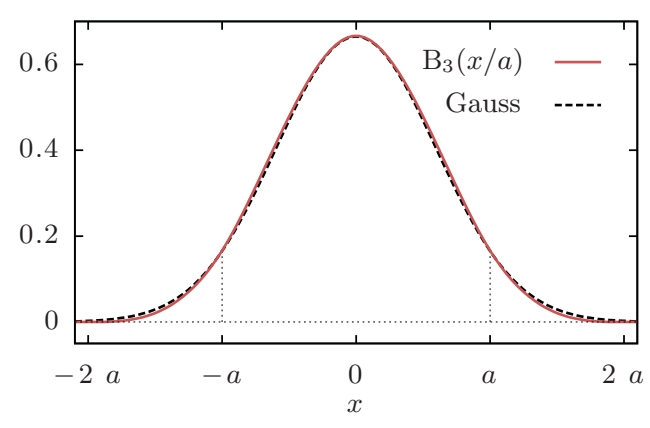

Figure 2. $B_{3}$ and Gaussian $(\sigma=0.6)$ smoothing kernels.

galaxies. In addition, we removed the small-scale finger-of-god redshift distortions to increase the resolution of the density fields (see e.g. Liivamägi et al. 2012)

Luminosity and filament probability density. Density fields are calculated on a Cartesian grid using kernel smoothing. We chose $B_{3}$ spline function (Fig. 2) as the kernel:

$$
B_{3}(x)=\frac{|x-2|^{3}-4|x-1|^{3}+6|x|^{3}-4|x+1|^{3}+|x+2|^{3}}{12} .
$$

The $B_{3}$ spline is preferable to e.g. the Gaussian kernel for it is compact in space and therefore does not generate noise in the low density regions as the latter does. We chose smoothing radius to be $a=1 h^{-1} \mathrm{Mpc}$ as this is the scale we expect to be relevant with respect to the effect. Density and probability field grid cell length is also set to $\Delta=1 h^{-1} \mathrm{Mpc}$. We don't use the filament spines presented in the (Tempel et al. 2014) catalogue but rather the raw filament probability fields (visit maps) which should offer more continuous representation of the filamentary environment.

We will calibrate the method by performing similar analysis on N-body simulations containing galaxies, dark matter and gas and test our estimates against existing observations. This will be studied in detail in the upcoming paper by Nevalainen et al. (in prep).

\section{Results}

Line of sight analysis. We present the results of the preliminary analysis. The set of limiting parameters used is given in Table 1 . We require our structures to have a minimum length to increase the chance of having enough column density. As the WHIM gas temperature has to be in certain interval, it is also reasonable to set both lower and upper limits to the density field values. For the filament probability we require the field value at the vertice to be above 0.05 , which corresponds to the $95 \%$ probability of being in the filament. The plane of sky is sampled uniformly with lines of sight and following attributes are calculated: the count of continuous structures, the total length covered

Table 1. Parameters for the line of sight analysis.

\begin{tabular}{|l|c|c|c|}
\hline Field type & Min density & Max density & Minimum length \\
\hline $\begin{array}{l}\text { luminosity } \\
\text { density }\end{array}$ & $\begin{array}{c}0.5 \\
\text { mean density }\end{array}$ & $\begin{array}{c}10.0 \\
\text { mean density }\end{array}$ & $\begin{array}{c}3 \\
h^{-1} \mathrm{Mpc}\end{array}$ \\
\hline $\begin{array}{l}\text { filament } \\
\text { probability }\end{array}$ & 5 & - & $\begin{array}{c}3 \\
h^{-1} \mathrm{Mpc}\end{array}$ \\
\hline
\end{tabular}

Notes:

1 Mean luminosity density value for the SDSS DR8 is $1.667 \cdot 10^{-2}$. 

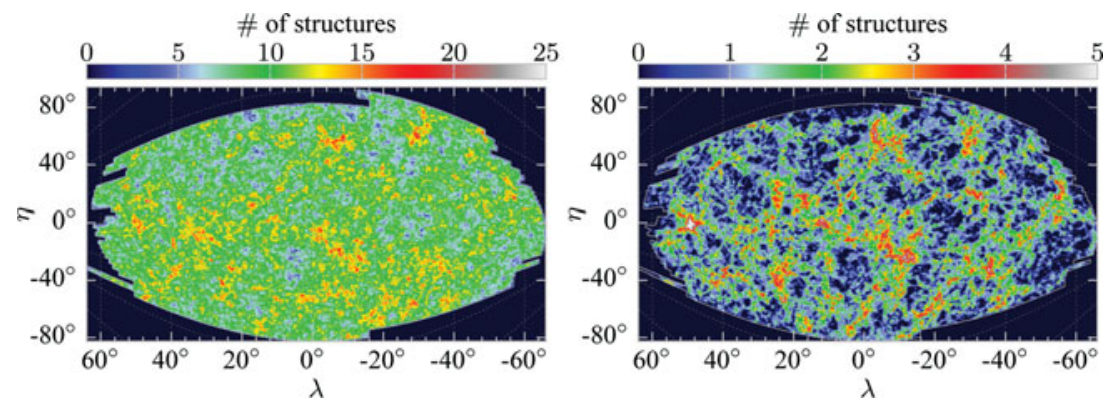

Figure 3. Number of structures along the lines of sight along the line of sight in the luminosity density field (left) and in the filament probability field (right).
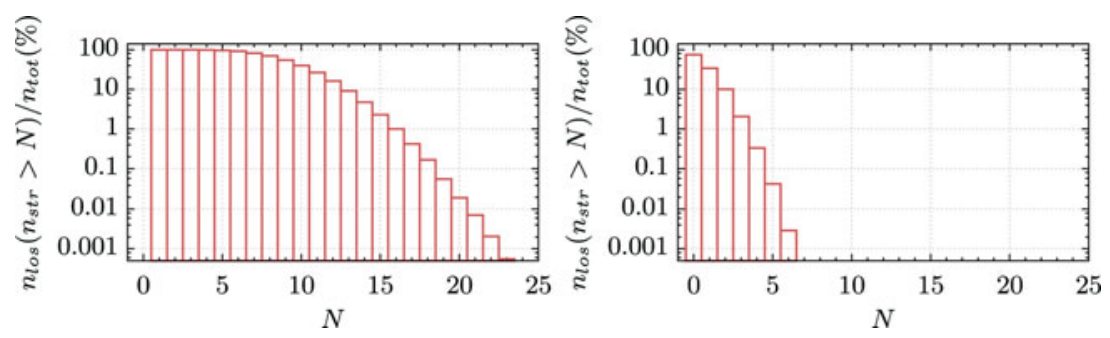

Figure 4. Distribution of the amount of structures along the lines of sight in the luminosity density field (left) and in the filament probability field (right).

by structures, and in case of luminosity density, the cumulative column density along the line of sight. Due to room limitations, we present here only as example the analysis of number of structures. Fig. 3 shows the sky distribution of the structure counts in both fields. We see that all lines of sight have at least a few structure on them, however there are much less of those with high number. The number of filament structures is significantly smaller and fair amount of directions do not cross any filament. This is also confirmed by the distortion curves on Fig. 4.

Conclusion. We have found that lines of sight with notable number of suitable structures are sparse. Therefore, a careful analysis of foregrounds is necessary e.g. before applying for time for time-consuming observations. Sky maps of projected density are useful tool to be included in the study when estimating the likelyhood for detection of WHIM.

\section{References}

Aihara, H. et al. 2011, ApJ, 29, 193

Buote, D. A., Zappacosta, L., Fang, T., Humphrey, P. J., Gastaldello, F., \& Tagliaferri, G. 2009, Science, 695, 1351

Cen, R. \& Ostriker, J. P. 2006, Science, 650, 560

Cui, W., Baldi, M., \& Borgani, S. 2012, MNRAS, 424, 993

Liivamägi, L. J., Tempel, E., \& Saar, E. 2012, A\& A, 539, A80

Martínez, V. J. \& Saar, E. 2003, Statistics of the Galaxy Distribution (Chapman \& Hall/CRC, Boca Raton)

Nicastro, F., Mathur, S., \& Elvis, M. 2008, Science, 319, 55

Tempel, E., Tago, E., \& Liivamägi, L. J. 2012, A\&A, 540, A106

Tempel, E., Stoica, R. S., Martínez, V. J., Liivamägi, L. J., Castellan, G., \& Saar, E. 2014, MNRAS, 438, 3465 